\title{
EVALUATION OF AIRBORNE IMAGE VELOCIMETRY APPROACHES USING LOW-COST UAVS IN RIVERINE ENVIRONMENTS
}

\author{
F. Ioli ${ }^{1, *}$, L. Pinto ${ }^{1}$, D. Passoni ${ }^{1}$, V. Nova ${ }^{1}$, M. Detert $^{2}$ \\ ${ }^{1}$ Department of Civil and Environmental Engineering (DICA), Politecnico di Milano - francesco.ioli@ mail.polimit.it, \\ (livio.pinto, daniele.passoni, valentina.nova)@polimi.it \\ ${ }^{2}$ Laboratory of Hydraulics, Hydrology and Glaciology (VAW), ETH Zürich - detert@ vaw.baug.ethz.ch
}

\section{Commission II, WG II/5}

KEY WORDS: AIV, PIV, UAV, river surface velocity, image-based velocimetry, BASESURV, Fudaa-LSPIV, RIVeR

\begin{abstract}
:
Traditional flow velocity measurements in natural environments require contact with the fluid and are usually costly, time-consuming and, sometimes, even dangerous. Particle Image Velocimetry allows the flow velocity field to be remotely characterized from the shift of intensity patterns of sub-image areas in at least two video frames with a known time lag. Recently, Airborne Image Velocimetry has enabled the surface velocity field of large-scale water bodies to be determined by applying Particle Image Velocimetry on videos recorded by cameras mounted on unmanned aerial vehicles. This work presents a comparison of three Airborne Image Velocimetry approaches: BASESURV, Fudaa-LSPIV and RIVeR. For the evaluation, two nadiral videos were acquired with a low-cost quadcopter. The first was recorded under low flow and seeded conditions, the second during a flood event. According to the results obtained, BASESURV is an accurate and complete research oriented approach but it is time-consuming and neither a graphical interface nor documentation are yet provided. Fudaa-LSPIV is a well-developed software package, with a user-friendly graphical interface and good documentation. However it lacks some features and the source code is closed. RIVeR may be suitable for real time monitoring thanks to the rectification of velocity vectors only. Overall, all the codes are found to be effective in performing Airborne Image Velocimetry in riverine environments.
\end{abstract}

\section{INTRODUCTION}

Measuring flow velocities is one of the main issues of hydraulic engineering. Traditional flow measurements require contact with the fluid and are usually costly, time-consuming and, sometimes, even dangerous. Since the nineteen eighties, an image-based technique called Particle Image Velocimetry (PIV) has been widely used to remotely capture the whole velocity field of a fluid (Adrian, 1991; Raffel et al., 1998). In PIV, velocity vectors are computed from the shift of characteristic intensity patterns of sub-image areas, called Interrogation Areas (IAs), in at least two video frames with a known time lag. For each IA in the first frame, a local integer displacement vector is computed by evaluating the cross-correlation with the corresponding IA in the second frame (Keane and Adrian, 1992). The integer displacement may then be refined by using a sub-pixel estimation technique (Nobach et al., 2005). Instantaneous flow velocity fields are derived by dividing the displacement vectors by the time lag.

PIV has been successfully used in laboratories over the past 35 years (Adrian, 2005). More recently, this technique has been adapted to measure surface velocities of largescale water bodies and, therefore, it has been labelled as Large-Scale Particle Image Velocimetry (LSPIV) (Fujita et al., 1998). LSPIV has been successfully applied adding artificial tracers to the flow (Jodeau et al., 2008; Detert and Weitbrecht, 2015; Detert et al., 2017). In naturally seeded or unseeded conditions LSPIV measurements may be carried out by recognising on the images floating foam, suspended sediments or specular reflections on the free surface deformations (Fujita and Hino, 2003; Muste et al., 2008).

\footnotetext{
${ }^{*}$ Corresponding author
}

Many authors have assessed the ability of unseeded LSPIV of characterizing surface velocities under high flow regimes, where the turbulent structures advected by the main flow are the dominant surface features (Creutin et al., 2003; Fujita and Hino, 2003; Le Coz et al., 2010; Fujita and Kunita, 2011). On the other hand, surface velocity determination under low flow regimes in absence of floating materials is more challenging because LSPIV identifies also capillary-gravity waves, which travel with their own speed and direction (Benetazzo et al., 2017).

In LSPIV, the camera was traditionally required to be fixed at an elevated position with respect to the river. However, the possibility of performing the analysis by flying or hovering above the river provides more degrees of freedom and flexibility. Airborne Image Velocimetry (AIV) was first developed in Japan by Fujita and Hino (2003) and Fujita and Kunita (2011), who used images taken from a helicopter to compute the surface velocity of a river under both low flow and flood conditions. First AIV experiments using Unmanned Aerial Vehicles (UAVs) were conducted by Fujita et al. (2015) and Detert and Weitbrecht (2015). Those were mostly focused on video stabilization by using projective transformations and on PIV. Recently, Detert et al. (2017) have developed a full proof-of-concept for low-cost AIV by using Structure from Motion (SfM) techniques to obtain the orthophotos to be used for velocimetry and discharge estimation. A similar approach was used by Detert et al. (2019) to measure a large surface velocity field in proximity to a hydropower plant in Switzerland. In both the studies, the cost of the equipment was less than $€ 2000$.

Although the hovering performance of recent UAVs have 
improved in the past few years, the wind gusts still introduce some shaking effects in the recorded videos. These must be corrected before applying PIV in order to avoid spurious vectors due to the apparent movement of the images. Video stabilization mainly consists in warping the frames by applying a geometric transformation, usually a projective transformation, estimated on the basis of double points detected on pairs of video frames (Fujita et al., 2015; Detert and Weitbrecht, 2015). Then, frames have to be rectified in order to correct the perspective effect due to the camera central projection and to provide metric information to the pixels. To this end, a transformation between the 3D world reference system and the 2D image reference system has to be estimated. Most common approaches assume the water surface as a plane and estimate a 2D-to-2D projective transformation (Fujita et al., 1998; Jodeau et al., 2008). Alternatively, a 3Dto-2D photogrammetric relation, such as the Direct Linear Transformation (DLT), may be employed (Muste et al., 2008). Under the assumption that the video is properly stabilized, only one geometric transformation between the world and the image reference systems can be estimated and applied to all the frames. Otherwise, a different transformation for each video frame has to be estimated.

Once the video is rectified, PIV is applied on each image pair to compute instantaneous flow velocity fields. In natural environments a large number of spurious velocity vectors generally result from PIV. These should be identified and filtered out in order not to have biased results. Finally, the sequence of instantaneous velocity fields may be averaged to compute a time-averaged surface velocity field.

In recent years, several studies on PIV applications in riverine environments have been developed, but just a small number of AIV software packages have been released to the public. In this work, a comprehensive overview over the available AIV approaches BASESURV, Fudaa-LSPIV and RIVeR is presented. A comparison between them is carried out in order to evaluate their relative performance in computing the surface velocity of a river using low-cost UAVs, under different flow conditions and both with and without tracer particles.

\section{OVERVIEW OF CURRENT AIV APPROACHES}

\subsection{BASESURV}

BASESURV (acronym that stands for BASic Environment for SURface Velocity computation) is a research-oriented software developed by M. Detert at the Laboratory of Hydraulics, Hydrology and Glaciology (VAW) at ETH Zürich. It is written in MATLAB $^{\circledR}$, but the code it is not released to the public. Neither a GUI nor documentation are yet available. For PIV, BASESURV is based on the code taken from PIVlab 1.42, developed by W. Thielicke (Thielicke and Stamhuis, 2014). Distinctive features of BASESURV are the orthorectification approach and the post-processing filters of spurious vectors. The former consists in transforming all the video frames by using a projective transformation between each of them and an orthophoto of the area. The latter is based on a dispersion analysis of the time-series of the velocity components at each point of the grid.

\subsection{Fudaa-LSPIV}

Fudaa-LSPIV is a software package developed by M. Jodeau, A. Hauet, J. Le Coz and co-workers. Its development has been executed by DeltaCAD since 2010 with financing and under the direction of EDF and Irstea (France) (Jodeau et al., 2019). The version used in this work is Fudaa-LSPIV 1.7.1, released on 03/06/2019. Fudaa-LSPIV has a Java GUI, which calls executables written in Fortran, and it is diffused to the public freely under GPL licence. The software is equipped with a good user manual that provides detailed explanations on the algorithms implemented. This, together with the user-friendly graphical interface, makes Fudaa-LSPIV easy to be used.

\subsection{RIVeR}

RIVeR (Rectification of Image Velocity Results) is an application developed by A. Patalano and co-workers in the Center for Water Research and Technology (CETA) at the National University of Cordoba, Argentina, in 2013. It is written in MATLAB $^{\circledR}$ and released to be used free of charge with MATLAB ${ }^{\circledR}$ Runtime 8.5. At the time of this work, the latest version of RIVeR is 2.4 .3 , released on $13 / 11 / 2019$. Similarly to BASESURV, RIVeR is based on PIVlab 2.31 for the PIV part. The user manual helps in using the software, yet it does not provide any detail on the algorithms. The aim of RIVeR is to provide a fast approach in large-scale water surface characterization. In fact, it does not rectify all the video frames, but it performs PIV on the raw images and then rectifies the velocity vectors afterwards. It was initially designed for processing videos or images taken from an oblique view (Patalano et al., 2017).

\section{EXPERIMENT SETUP}

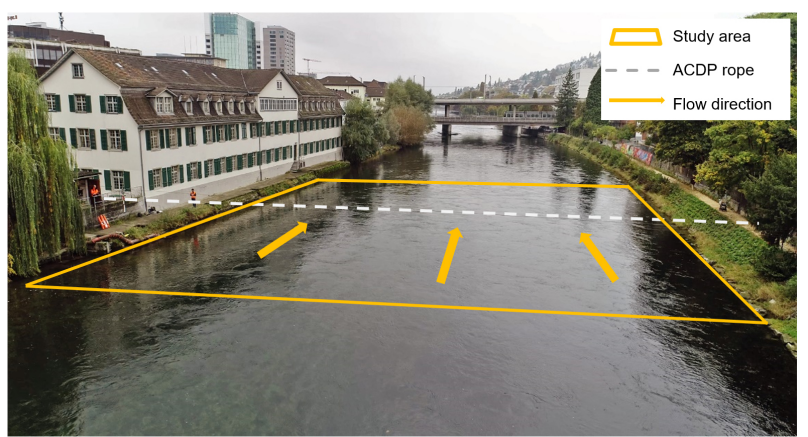

Figure 1. Limmat - Zürich Unterhard gauging station. A rope is suspended across the river to control monitoring instruments.

For AIV approaches comparison, two datasets, called dataset A and dataset $\mathrm{B}$, were acquired at the Zürich Unterhard gauging station on river Limmat (Switzerland) (Figure 1). Dataset A was acquired under stable flow conditions, with a discharge comparable to the October daily average (period 1938-2018). To obtain a significant greyscale contrast on the frames, the flow was seeded with tracers from an upstream bridge: $\sim 2 \mathrm{~m}^{3}$ of $100 \%$ biodegradable corn starch chips MEDEWO FILL-PAC Bio, with diameter of $\sim 3 \mathrm{~cm}$, were used. Immediately before the acquisition of video A, additional flow measurements were carried out by the Swiss Federal Office for the Environment (FOEN) with a hydrometric impeller. Dataset B was acquired when the discharge was comparable to the 1-year return period flood event and no tracers were used. Both the videos were acquired with a nadiral viewing geometry. The main characteristics of the two datasets are summarized in Table 1.

The videos were acquired with a quadcopter UAV DJI Phantom 4 Pro+. This was equipped with an on-board camera with a 


\begin{tabular}{ccccc}
\hline Name & Date & $\begin{array}{c}\text { Discharge } \\
{\left[\mathrm{m}^{3} \mathrm{~s}^{-1}\right]}\end{array}$ & $\begin{array}{c}\text { Water level } \\
{[\mathrm{m} \text { a.s.1. }]}\end{array}$ & Seeding \\
\hline A & $22 / 10 / 19$ & 83 & 400.2 & Yes \\
B & $10 / 10 / 19$ & 220 & 401.2 & No \\
\hline
\end{tabular}

Table 1. Summary of the characteristics of the two datasets.

$1^{\prime \prime}$ CMOS sensor, mounted on a three-axis gimbal. The focal length was $8.8 \mathrm{~mm}$ and the image size was $4096 \times 2160 \mathrm{px}$. The take-off weight of the UAV was $1.4 \mathrm{~kg}$ and the total cost of quad-copter and accessories was below 2,000€ (March 2018). Video A was $35 \mathrm{~s}$ long and was acquired with a frame rate of $29.97 \mathrm{~Hz}$ (1048 frames); video B had a duration of $30 \mathrm{~s}$ and a framerate of $23.97 \mathrm{~Hz}$ (718 frames).

The topographic survey was conducted within the Swiss national reference system CH 1903+/LV95. This was materialized with 29 Ground Control Points (GCPs) placed on natural elements along the riparian sides of the river. Their coordinates were acquired by Detert and co-workers on $10 / 10 / 2018$ by using a GNSS Trimble R8 in RTK. From a video acquired with the UAV DJI Phantom 4 Pro+ on the same day, 50 nadiral images were used to build a 3D photogrammetric model by using the SfM software Agisoft Metashape Professional. The global RMSE of the model, computed by using 9 check points, was equal to $0.12 \mathrm{~m}$. The $\mathrm{SfM}$ model was used to extract an orthophoto and a Digital Surface model (DSM), both with a Ground Sample Distance (GSD) of $0.05 \mathrm{~m} \mathrm{px}^{-1}$. From these, sets of artificial GCPs were extracted in order to georeference video A and video B. By considering the geometrical accuracy of the model and the GSD, the artificial GCPs accuracy was in the order of magnitude of the decimetre.

\section{PROCESSING OF THE VIDEOS}

\subsection{Video stabilization and rectification}

Before video stabilization and rectification, the camera should be calibrated to reduce the distortions induced by the lenses. In BASESURV and RIVeR, this may be performed estimating the intrinsic camera parameters through the MATLAB ${ }^{\circledR}$ Camera Calibrator app, available in the Computer Vision Toolbox ${ }^{\mathrm{TM}}$. On the contrary, Fudaa-LSPIV does not allow camera calibration.

The steps of video stabilization and rectification are crucial for AIV. In BASESURV these were performed simultaneously, by estimating a homography transformation between each Video Frame (VF) and a orthorectifed Reference Frame (RF). The latter was a video frame which had been previously rectified over an orthophoto with a homography by manually collimating double points (RMS of the reprojection errors equal to $0.14 \mathrm{~m}$ for video A and $0.18 \mathrm{~m}$ for B). Subsequently, KAZE interesting operator (Alcantarilla et al., 2012) was employed to detect double points between each VF and the RF. Provided that the homography is a model for the central projection between two planes (i.e. the water surface and the image plane), the double points had to lay on the same plane as the water surface. Those not belonging to the water surface plane (e.g. trees and buildings) or those within moving areas (e.g. water flow and shadows) were rejected by a binary mask defined on the RF. Although it should be avoided, a portion of the buildings in the study area was not excluded by the binary mask in order to find enough double points in the south-west area (Figure 2). Hence, the time-series of the 4 corners coordinates of the rectified frames were smoothed by a robust LOESS method (Cleveland, 1979) in order to reduce artificial errors in the rectification e.g. due to a little number of matching points in particular areas.

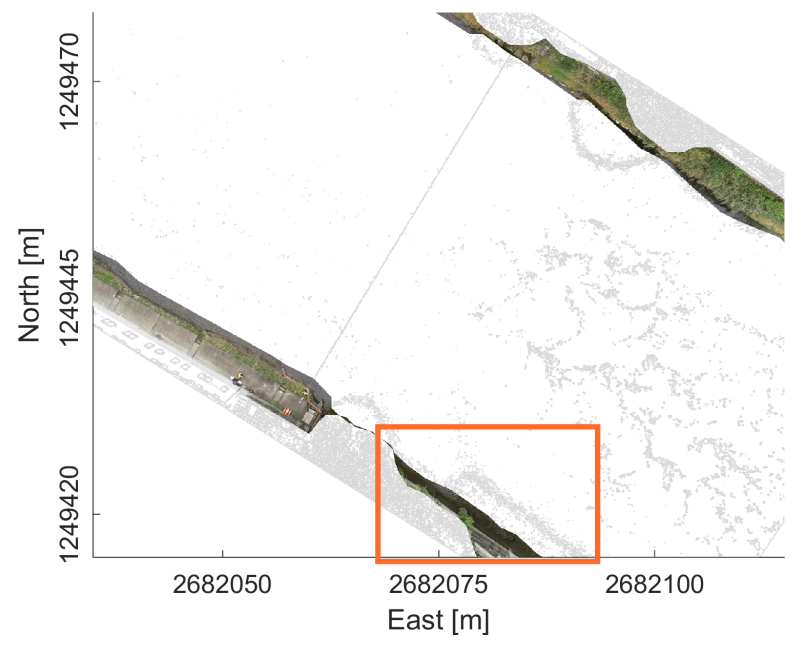

Figure 2. Binary mask applied on video A RF in BASESURV. The south-west area in which there are no points at the water surface level along the riparian side is marked in the box.

In Fudaa-LSPIV stabilization and rectification were executed separately. For the stabilization, a binary mask was defined over the flow area in the first video frame and applied to all the others. Homography transformations between each video frame and the first one were estimated by means of double points detected by the SURF operator (Bay et al., 2008). For the image rectification in Fudaa-LSPIV, a set of GCPs, manually collimated on the first video frame, was used to estimate the DLT parameters between the image and the world reference system. The transformation was then applied to all the video frames. This approach required that the video had been properly stabilized beforehand. The RMS of reprojection errors of the GCPs was $0.05 \mathrm{~m}$ for both video A and B. The implementation of DLT, instead of homography, might have been the reason of the lower RMSE in Fudaa-LSPIV than in BASESRUV.

In RIVeR, video stabilization was implemented by linking each video frame to the previous one with an affine transformation, estimated on the basis of matching points detected by FAST (Rosten and Drummond, 2005). This stabilization approach was not optimal because of drift error, cumulating over the video. In order to exclude moving areas from the matching point research, a mask over the flow area was defined. RIVeR did not rectify all the images, but only the velocity vectors computed by PIV over the stabilized video frames. Similarly as Fudaa-LSPIV image rectification, the vector rectification in RIVeR was performed with DLT and a set of GCPs, manually collimated on the first video frame. However, RIVeR did not implement any tool for evaluating the quality of the estimated transformation.

\subsection{Image pre-processing}

Various image enhancing techniques are typically applied before PIV to improve the result of the correlation (Raffel et al., 2018). As they were both based on PIVlab, BASESURV and RIVeR adopt almost the same tools. On the contrary, this processing step was completely absent in Fudaa-LSPIV. In BASESURV and RIVeR, the background, computed as the average of all the video frames, was subtracted to all the images 
in order to enhance the moving particles only. Moreover, a noisy pixel intensity capping was applied in BASESURV: for video A, pixels with greyscale intensity lower than $10 \%$ of the highest radiometric value, which were likely not related to seeding particles, were set to zero. For video B, where no clear signal was present, a threshold of $1 \%$ was applied.

\subsection{PIV}

In BASESURV, PIV was performed on a sequence of orthophotos previously rectified. For each image pair, the cross-correlation between an IA on the first frame and the corresponding one in the second was carried out in the frequency domain, by means of Fast Fourier Transform (FFT). A three steps iterative multi-pass approach with deforming window was applied (Thielicke and Stamhuis, 2014; Scarano and Riethmuller, 1999; Scarano, 2002). Cross-correlation was applied three times on the same area and the integer displacement estimated in the first pass was used to offset the IA in the following ones. At each pass, the size of the IA was gradually reduced by factor 2 in order to achieve a finer spatial resolution. Table 2 shows the size of the IAs employed to process video A and B. The integer displacement obtained by correlation was refined with a 3-point Gaussian sub-pixel estimation technique.

In Fudaa-LSPIV, the cross-correlation analysis was performed in the spatial domain. For every grid point in the first image, the correlation coefficient between the IA centred on it and the corresponding IA in the second image was computed. The calculation was performed only for points within a search area defined on the second image to save computational time (Jodeau et al., 2019). Since Fudaa did not implement any iterative multi-pass approach, IAs comparable to the final ones of BASESURV were used (Table 2). The sub-pixel estimation by fitting two unidimensional Gaussian distributions on the correlation matrix. In order to have consistent results between Fudaa-LSPIV and BASESURV, the same grid of points were used in both the approaches.

Since both RIVeR and BASESURV are based on PIVlab, they implement similar PIV algorithms. In RIVeR, however, PIV was applied on a sequence of non-orthorectified images. For each image pair, cross-correlation was computed in the frequency domain with FFT, by using 3 passes with deforming windows and a 3-point Gaussian sub-pixel estimation technique. For both dataset $\mathrm{A}$ and $\mathrm{B}$, the same size of IAs as in BASESURV were employed. Nevertheless, the grid used in RIVeR was different from the others because it was defined on the non-orthorectified frames and transformed afterwards.

The time lag $\Delta t$ between each pair of images is a central issue in PIV. In BASESURV, it can be arbitrary chosen by deciding the most suitable interval in terms of number of frames $\left(n_{f}\right)$. Assuming for instance $n_{f}=4$, the frames are processed as follows: $[(1,5),(2,6),(3,7), \ldots,(N-4, N)]$, where $N$ is the total number of frames in the video. This approach allows for a large enough time lag to reduce errors due to small particle displacements, but using at the same time the whole amount of data in the video. In both Fudaa-LSPIV and RIVeR, the time lag must be chosen during the frame extraction from the raw video, by extracting one in every $n_{f}$ frame. Hence, the processing sequence would be $[(1,5),(5,9),(9,13), \ldots,(N-4, N)]$ and just a small subset of the available information is used. For video $\mathrm{A}, n_{f}=4$ was taken: this was a fair compromise between a sufficient time lag, $\Delta t=0.133 \mathrm{~s}$, and a large enough pairs

\begin{tabular}{ccccccc}
\hline Vid & SW & $\begin{array}{c}n_{f} \\
{[-]}\end{array}$ & $\begin{array}{c}\Delta t \\
{[\mathrm{~s}]}\end{array}$ & $\begin{array}{c}n_{t} \\
{[-]}\end{array}$ & $\begin{array}{c}\text { IA }_{i n} \\
{[\mathrm{px}]}\end{array}$ & $\begin{array}{c}\text { Passes } \\
{[-]}\end{array}$ \\
\hline \multirow{4}{*}{ A } & BSV & 4 & 0.133 & 1048 & $128 \times 128$ & 3 \\
& Fud & 4 & 0.133 & 262 & $32 \times 32$ & 1 \\
& RIV & 4 & 0.133 & 262 & $128 \times 128$ & 3 \\
\hline \multirow{4}{*}{ B } & BSV & 1 & 0.042 & 718 & $256 \times 256$ & 3 \\
& Fud & 1 & 0.042 & 718 & $64 \times 64$ & 1 \\
& RIV & 1 & 0.042 & 718 & $256 \times 256$ & 3 \\
& BSV & 12 & 0.501 & 718 & $256 \times 256$ & 3 \\
\hline
\end{tabular}

Table 2. PIV parameters used in BASESURV (BSV),

Fudaa-LSPIV (Fud) and RIVeR (RIV). Column $n_{f}$ refers to the interval in terms of number of frames within every pair of images used for PIV; $n_{t}$ is number of instantaneous results.

of frames available $n_{t}$ in Fudaa-LSPIV and RIVeR (Table 2). For video B, because of some problems in the stabilization in Fudaa-LSPIV, it was necessary to use all the video frames and $\Delta t=0.042 \mathrm{~s}$ in order to have an even comparison between the three AIV approaches. Several stabilization tests using other frame interval than $n_{f}=1$ in Fudaa-LSPIV, in fact, were collapsing and it was not possible to proceed with the further steps of the AIV workflow. However, the time lag in PIV is strongly related to the lowest resolvable velocity (Scarano and Riethmuller, 1999) and a $\Delta t=0.042 \mathrm{~s}$ was too small for the expected velocity range in river Limmat $\left(0.1 \mathrm{~m} \mathrm{~s}^{-1}\right.$ to $\left.3 \mathrm{~m} \mathrm{~s}^{-1}\right)$. Therefore, video B was further processed with a larger time lag $\Delta t=0.501 \mathrm{~s}$ in order to have a more robust PIV analysis.

\subsection{Filtering of spurious vectors}

All of the three AIV approaches allow the spurious vectors in each instantaneous velocity field to be filtered, e.g. by defining lower and upper thresholds for each velocity components or with the normalized median test (Westerweel and Scarano, 2005). In addition to those, in Fudaa-LSPIV it is possible to exclude the vectors with a low correlation index computed by PIV (Jodeau et al., 2019). Only BASESURV implements an effective filtering tool based on a dispersion analysis of the time-series of the velocity components at each point of the grid. This assumes that the time-series of $u$ and $v$, in the $u$ - $v$ plane, follows a bivariate Gaussian distribution. In order to separate the inlier vectors from the outliers, two 2D multivariate Gaussian models are fitted on the sample built by considering the whole time-series of velocity vectors at each grid point and the surrounding eights together. Points belonging to the more sparse Gaussian model are considered as spurious vectors and rejected. The time series of the inlier vectors, accepted during the previous step, are finally smoothed by applying a robust LOESS smoothing (Cleveland, 1979).

\subsection{Computation of the time-averaged velocity fields}

For each point on the grid, the mean or the median velocity vector may be computed from the time-series. The latter is known to be more robust against the outliers. BASESURV employs the median operator, whilst both Fudaa-LPSIV and RIVeR use the mean. Nevertheless, Fudaa-LSPIV enables all the computed instantaneous velocity fields to be exported and processed with an external software. The median velocity field was therefore computed in MATLAB ${ }^{\circledR}$, in addition to the average one. On the contrary, RIVeR does not allow for further processing of the instantaneous results. 


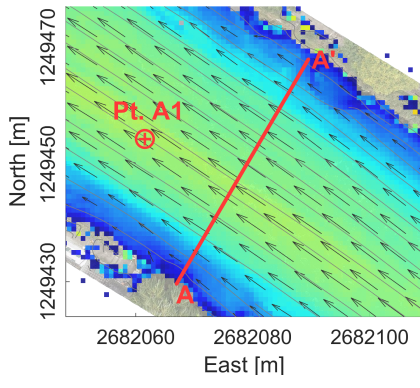

(a) BASESURV (median)

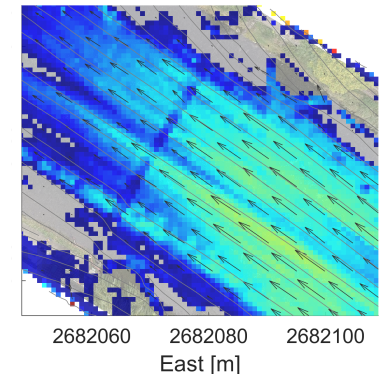

(b) Fudaa-LSPIV (average)

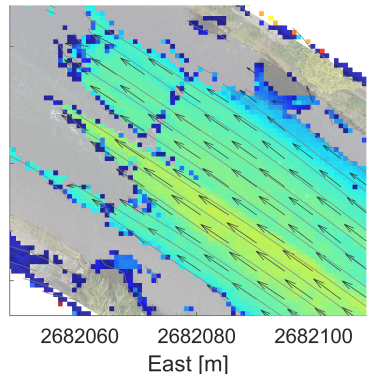

(c) Fudaa-LSPIV (median)

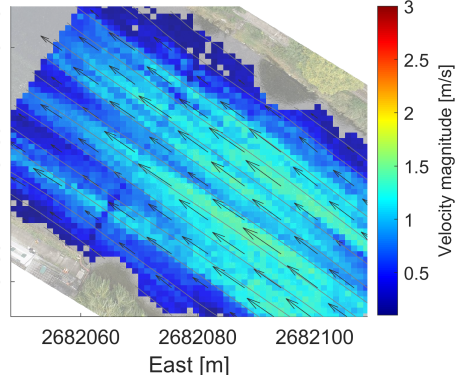

(d) RIVeR (average)

Figure 3. Time-averaged SVFs computed from dataset A: (a) BASESURV (median); (b) Fudaa-LSPIV (average); (c) Fudaa-LSPIV (median); (d) RIVeR (average). In (a) the location of the grid point Pt. A1 (Figure 4) and cross-section AA' (Figure 5) are marked.

\section{RESULTS OF THE COMPARISON}

\subsection{Dataset A}

Figure 3 illustrates the time averaged Surface Velocity Fields (SVFs) obtained from dataset A with the three AIV approaches. In general, the flow is homogeneous with surface velocities ranging from $0.6 \mathrm{~m} \mathrm{~s}^{-1}$ near the riparian sides to $1.7 \mathrm{~m} \mathrm{~s}^{-1}$ in the centre. The low-flow and steady conditions, in combination with the intensity capping filters (see Section 4.2), made it challenging to compute velocity vectors without any seeding particles within the IAs. The SVFs obtained with Fudaa-LSPIV and RIVeR (Figures $3 \mathrm{~b}$ and $3 \mathrm{~d}$ ) show an underestimation of the velocities compared to that of BASESURV (Figure 3a), especially in the downstream area and in the central-upstream part of the river. Figure 4 shows the time-series of velocity vectors at Pt. A1. In Figures $4 \mathrm{~b}$ and $4 \mathrm{c}$, a cluster of nearzero vectors is predominant during the first $\sim 15 \mathrm{~s}$, before the arrival of the first tracers within the camera view. Further clusters can be found in few other portions of the video, probably due to a non-perfectly homogeneous seeding. In fact,
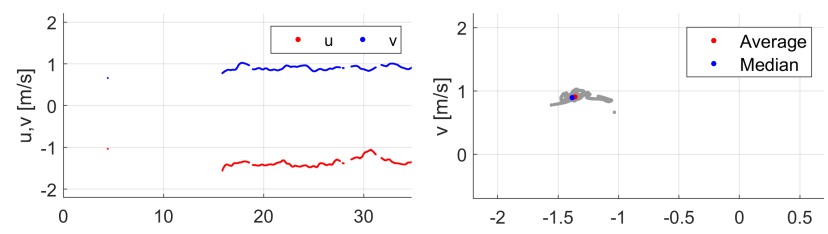

(a) BASESURV
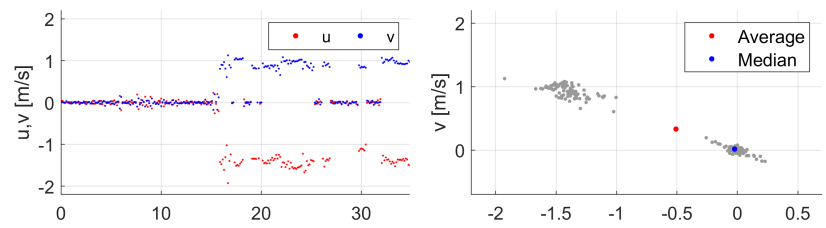

(b) Fudaa-LSPIV
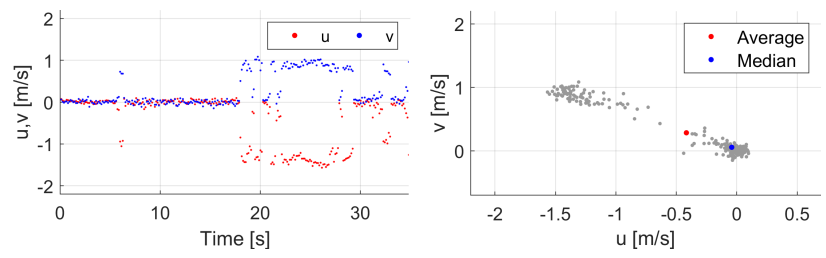

(c) RIVeR

Figure 4. Time-series and scatter plots of the two Cartesian velocity components $u$ and $v$ at grid point Pt. A1. The location of Pt. A1 is given in Figure 3a. when no clear signal was detectable by cross-correlation, the residual orthorectification errors in Fudaa-LSPIV and RIVeR produced low-velocity spurious vectors, which might have caused the low-bias in the time-averaged SVFs. This lowbias effect is more relevant in the time-averaged SVF if the mean operator is employed rather than the median (Figure 3c). Nevertheless, if the amount of near-zero vectors in the timeseries is greater than $50 \%$, the median vector falls into the low-value cluster and it is filtered out using thresholds on the minimum and maximum accepted velocity magnitude, producing a no-data result in the final velocity field (Figures $4 \mathrm{~b}$ and $4 \mathrm{c}$ ). The 2D multi Gaussian post-processing filters implemented BASESURV (see Section 4.4) are able to identify and remove these outlier clusters (Figure 4a). The very low dispersion of the BASESURV time series and its deterministic appearance is due to the RLOESS smoothing filter. Moreover, the time-series obtained with BASESURV are more dense than those obtained with Fudaa-LSPIV and RIVeR due to the higher number of instantaneous results (see Table 2).

Both the Fudaa-LSPIV and RIVeR time-averaged SVF are characterized by noise in proximity to the ADCP rope. As it was $\sim 2 \mathrm{~m}$ above the water surface, during frame rectification the rope was mapped in different positions, depending on the position and attitude of the camera. Therefore spurious vectors, mostly characterized by low velocity values and high scattering in the direction, were computed by PIV. Only the BASESURV post-processing filters were able to identify and exclude this cluster of outlier vectors.

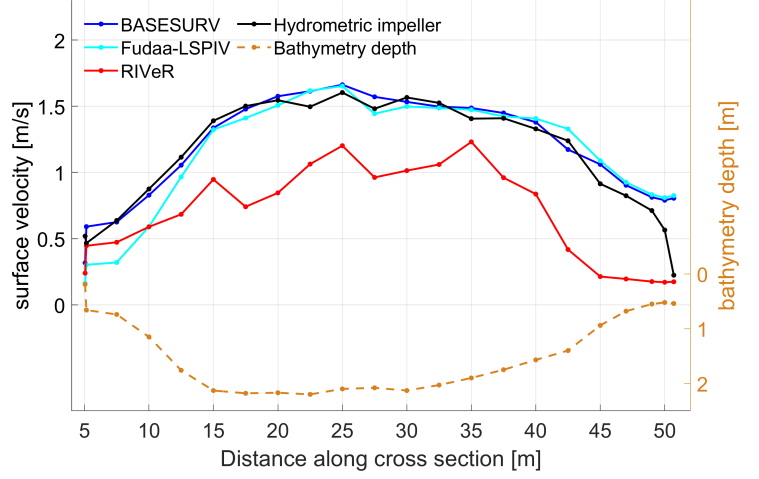

Figure 5. AIV velocity profiles along cross-section AA' (location in Figure 3a) compared to that measured by the hydrometric impeller. Missing data in AIV profiles were extrapolated according to the technique proposed by Le Coz et al. (2010).

In Figure 5, the velocity profiles measured by the hydrometric impeller $0.19 \mathrm{~m}$ below the water surface are compared against 
those obtained by the three AIV approaches along the crosssection AA'. For Fudaa-LSPIV, only the median SVF was considered. In the centre of the $45 \mathrm{~m}$ wide river, i.e. after $5 \mathrm{~m}$ from each riparian side, relative percentage errors in the estimated velocity of $4 \%, 5 \%, 36 \%$ were obtained respectively with BASESURV, Fudaa-LSPIV and RIVeR. Higher errors, respectively, $56 \%, 71 \%, 47 \%$, were obtained with all the approaches within $5 \mathrm{~m}$ from the riparian sides.

\subsection{Dataset B}

Figure 6 shows the time-averaged SVFs obtained from video B. The surface velocities range between $1.5 \mathrm{~m} \mathrm{~s}^{-1}$ near the riparian sides and $2.5 \mathrm{~m} \mathrm{~s}^{-1}$ in the central part of the flow. As explained in Section 4.3, video B was processed with a short time lag $\Delta t=0.042 \mathrm{~s}$ in order to have an even comparison between the three AIV approaches. As there were not significant differences between the mean and median timeaveraged SVF computed by Fudaa-LSPIV, only the first is presented. Figures 6a-c evidence similar time-averaged SVF, rather noisy on the right-hand side. On the left-hand side of the river, in fact, the flow was carrying a large amount of suspended solids that worked like natural seeding particles, improving PIV cross-correlation. On the right-hand side there were fewer patterns on the water surface. On the contrary, the BASESURV result with $\Delta t=0.501 \mathrm{~s}$ (Figure $6 \mathrm{~d}$ ) reveals a homogeneous and smooth SVF, with slightly higher velocities.
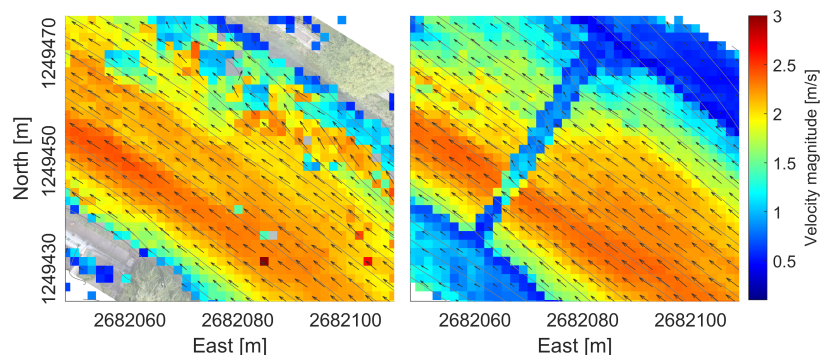

(a) BASESURV $\Delta t=0.042 \mathrm{~s}$

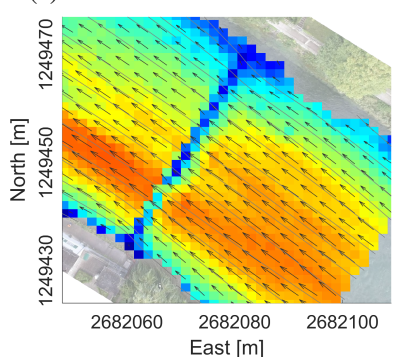

(b) Fudaa-LSPIV $\Delta t=0.042 \mathrm{~s}$

(c) RIVeR $\Delta t=0.042 \mathrm{~s}$

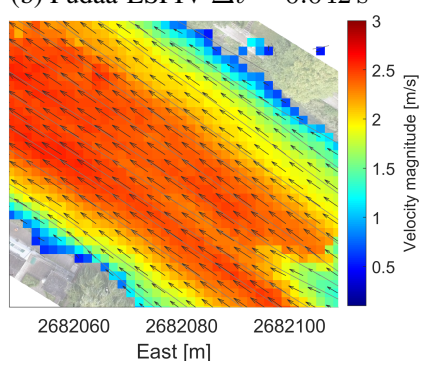

(d) BASESURV $\Delta t=0.501 \mathrm{~s}$

Figure 6. Time-averaged SVFs computed from dataset B. For each result, the PIV time lag $\Delta t$ is mentioned. (a) BASESURV ( $\Delta t=0.042 \mathrm{~s})$; (b) Fudaa-LSPIV ( $\Delta t=0.042 \mathrm{~s})$; (c) RIVeR $(\Delta t=0.042 \mathrm{~s}) ;(\mathrm{c}) \operatorname{BASESURV}(\Delta t=0.501 \mathrm{~s})$.

As the river did not present any perturbing agent nor changes in the bathymetry, the non-homogeneous velocities along the streamwise direction (Figures 6a-c) are symptoms of misestimated velocities due to a too short PIV time lag used rather than specific AIV software performance. This is mostly caused by sub-pixel estimation uncertainties. In fact, when the integer displacement estimated by cross-correlation is very small (often equal to just $1 \mathrm{px}$ in dataset B), errors in the subpixel estimation have a strong impact on the accuracy of the estimated velocity.

\begin{tabular}{|c|c|c|c|c|}
\hline \multicolumn{5}{|c|}{$\mathrm{Q}\left[\mathrm{m}^{3} \mathrm{~s}^{-1}\right]$} \\
\hline & BSV & Fud & RIV & BSV \\
\hline$\Delta t$ & $0.042 \mathrm{~s}$ & $0.042 \mathrm{~s}$ & $0.042 \mathrm{~s}$ & $0.501 \mathrm{~s}$ \\
\hline AIV & {$[170,184]$} & {$[176,192]$} & {$[174,190]$} & {$[210,228]$} \\
\hline $\mathrm{RC}$ & \multicolumn{4}{|c|}{$220 \pm 22$} \\
\hline
\end{tabular}

Table 3. Comparison between the AIV-based discharge against that estimated with the Rating Curve (RC) of river Limmat. The intervals of AIV discharge are due to the uncertainties in the

depth-averaged velocity evaluation (Le Coz et al., 2010).

As no in-situ velocity measurements were available for dataset $\mathrm{B}$, the assessment of the estimated velocity filed was carried out by means of the discharge. From the time-averaged SVFs, AIV-based discharge was obtained by numerical integration of the depth-averaged velocity over the bathymetry along a crosssection in proximity to the ADCP rope. The depth-averaged velocity was computed multiplying the free-surface velocity by a reduction coefficient ranging from 0.80 to 0.87 (Le Coz et al., 2010). Table 3 shows the comparison between the AIVbased discharge and that obtained with rating curve of the river Limmat. Only the discharge estimated by BASESURV with $\Delta t=0.501 \mathrm{~s}$ is comparable to the rating curve discharge.

\section{DISCUSSION}

Table 4 shows a summary of the computational time needed by each software package to process video A, normalized by the number of frames involved. The computations were carried out by a mid-range workstation with CPU Intel ${ }^{\circledR}$ Xenon $^{\circledR}$ E3-1245 v6 @ 3.7 GHz; RAM 16 GB; GPU Intel ${ }^{\circledR}$ HD Graphics P630.

\begin{tabular}{|c|c|c|c|}
\hline Software & Phase & $\begin{array}{c}\text { Time } \\
{[\mathrm{min} / \text { frame }]}\end{array}$ & $\begin{array}{l}\text { Time tot. } \\
\text { [min/frame] }\end{array}$ \\
\hline \multirow{3}{*}{ BSV } & Rectification $^{1}$ & 0.65 & \multirow{3}{*}{0.73} \\
\hline & PIV $^{2}$ & 0.06 & \\
\hline & Post-processing filters ${ }^{3}$ & 0.02 & \\
\hline \multirow{3}{*}{ Fud } & Video stabilization & 0.08 & \multirow{3}{*}{0.42} \\
\hline & Frame rectification & 0.23 & \\
\hline & PIV & 0.11 & \\
\hline \multirow{4}{*}{ RIV } & Frame undistortion & 0.06 & \multirow{4}{*}{0.16} \\
\hline & Video stabilization & 0.04 & \\
\hline & $\mathrm{PIV}^{4}$ & 0.06 & \\
\hline & Vector rectification & 0.004 & \\
\hline
\end{tabular}

Table 4. Comparison of the time needed by BASESURV (BSV), Fudaa-LSPIV (Fud) and RIVeR (RIV) to process video

A, normalized by the frames involved in the computation.

\subsection{Strengths and limitations of the AIV approaches}

The main strengths and limitations of the three AIV approaches are listed, grouped by the processing phase, as follows (Ioli, 2020):

6.1.1 Image undistortion: in contrast to BASESURV and RIVeR, Fudaa-LSPIV does not provide any tool to calibrate the camera. In RIVeR, video frame undistortion requires extremely long time to be performed. 
6.1.2 Stabilization and rectification: the different approach to rectification of BASESURV, Fudaa-LSPIV and RIVeR is crucial. Rectifying all the images is a more robust technique because each video frame may be independently rectified by estimating a specific geometric transformation. Moreover, state-of-the-art computer vision techniques may be applied e.g. to automatically detect the GCPs on the images by using a template matching technique, such as that implemented in the tool ImGRAFT (Messerli and Grinsted, 2015). The rectification of velocity vectors only allows for a computational time reduction. This may be particularly useful if the transformation between the image and the world reference system is known and does not change during the video, as with a fixed camera for real-time monitoring.

The approach proposed by BASESURV to stabilize and rectify the frames is probably the most accurate and robust. Only an orthophoto in which flat riparian sides are visible and a mask to exclude objects that does not belong to the water surface plane are required. If these are available, the process is completely autonomous. However, this approach is the most demanding in terms of computational time, almost twice that of Fudaa-LSPIV, if stabilization and rectification are summed together.

For the stabilization in Fudaa-LSPIV, out-of-plane objects should be excluded in the research of matching points when a projective transformation is used. Fudaa-LSPIV stabilization approach seems to be less robust than the others (Section 5.2). Stabilization in RIVeR is almost twice faster than that of FudaaLSPIV, but it is probably too approximate and may results in drift residual errors.

For image rectification in Fudaa-LSPIV and vector rectification in RIVeR, a minimum number of GCPs (or distances) are required to be measured in the field and collimated on one video frame. This may be time-consuming, both during the survey and in the processing phase. Moreover, RIVeR does not implement any tool for the accuracy assessment of the rectification.

6.1.3 Image pre-processing: Fudaa-LSPIV does not implement any image pre-processing tool. It would be useful to implement at least the background subtraction in future developments of the software.

6.1.4 PIV: as they are both based on PIVlab, BASESURV and RIVeR implement a more advanced and efficient correlation algorithm than that of Fudaa-LSPIV. The former works in the frequency domain with multiple passes and deforming windows. It is also almost twice faster than the algorithm implemented in Fudaa-LSPIV in the spatial domain.

The ability of BASESURV to use all the video frames in PIV computation, regardless of the time lag chosen, is a great advantage compared to the simple extraction of a subset of video frames. The best time lag can be chosen during the PIV phase without any loss of useful data.

6.1.5 Post-processing filters: all three approaches implement filters on each instantaneous velocity field independently. Only BASESURV implements effective filters based on the time-series of vectors at each grid point. However, it is challenging to develop smart post-processing filters.

The possibility to define elliptical region of acceptance in the $u-v$ plane, instead of a rectangular one based on thresholds, may be an improvement in instantaneous vectors filtering for FudaaLSPIV and RIVeR.
6.1.6 Time-averaged velocity field: the median operator may provide a more robust estimation of the time-averaged velocity against the outliers in both Fudaa-LSPIV and RIVeR.

\section{SUMMARY AND CONCLUSIONS}

The goal of this work was to carry out a comparison and evaluation of three AIV approaches: BASESURV, FudaaLSPIV and RIVeR. For the evaluation, two nadiral videos, called A and B, were acquired with a low-cost UAV. Video A was taken under monthly average discharge conditions and the flow was seeded with biodegradable corn starch chips. Video $\mathrm{B}$ was acquired during a 1-year return period flood and no tracers were used. Before the acquisition of video A, the surface velocity profile along a cross-section was measured with a hydrometric impeller as reference. In the centre of the $45 \mathrm{~m}$ wide river, i.e. after $5 \mathrm{~m}$ from each riparian side, relative percentage errors in the estimated velocity of $4 \%, 5 \%, 36 \%$ were obtained respectively with BASESURV, Fudaa-LSPIV (considering the median surface velocity field) and RIVeR. For video B, no in-situ velocity measurements were available, thus the flow discharge was estimated and compared to that measured by the gauging station as an assessment of the AIVbased surface velocity. A good agreement was found only when a suitable PIV time lag was employed.

According to the results obtained, BASESURV may be the best approach for research purposes: it is the most accurate and complete AIV approach. It is the only software implementing smart and effective post-processing filters based on the timeseries of vectors at each grid point. Moreover, it is the only one performing the video rectification by estimating one different geometric transformation for each video frame. However, neither a GUI nor a documentation are yet available and it is the most demanding software in terms of computational time.

Fudaa-LSPIV may be the best alternative for professional applications: it is a well developed AIV software package, with a user-friendly GUI and good documentation. The workflow is straight forward and it can be fully performed inside the software itself, from the video stabilization up to the time-averaged velocity field computation and, if needed, the discharge estimation. The whole AIV process is almost 1.7 times faster than that of BASESURV. However, the source code is closed and some useful tools are missing, such as image preprocessing or time-based post-processing filters.

RIVeR may be suitable for fast processing as well as for real time monitoring and alarm systems. The approach of RIVeR based on the rectification of the velocity vectors only is very effective in the reduction of computational time, especially when video stabilization is not needed, as with fixed cameras. In this case, the processing workflow might be almost 6 times faster than that of BASESURV.

Overall, all the AIV codes are found to be effective in performing AIV in riverine environments. Each enables the characterization of the surface velocity field without being in contact with the water, but analysing videos recorded by a camera mounted on low-cost UAVs. Future developments in AIV may involve a systematic investigation of the accuracy of this technique. This would expand the potential applications of AIV in both professional and scientific sectors. Moreover, the possibility to perform AIV analysis without neither GCPs nor seeding particles is an interesting outlook that may be explored in further studies. 


\section{ACKNOWLEDGEMENTS}

The flowmeter measurements by Jörg Hammer (FOEN) are gratefully acknowledged.

\section{REFERENCES}

Adrian, R.J., 1991. Particle-Imaging Techniques for Experimental Fluid Mechanics. Annual Review of Fluid Mechanics, 23(1), 261-304.

Adrian, R.J., 2005. Twenty years of particle image velocimetry. Experiments in Fluids, 39(2), 159-169.

Alcantarilla, P.F., Bartoli, A. and Davison, A.J., 2012. KAZE features. Computer Vision - ECCV 2012. Springer Berlin Heidelberg, 214-227.

Bay, H., Ess, A., Tuytelaars, T. and Van Gool, L., 2008. Speeded-Up Robust Features (SURF). Computer Vision and Image Understanding, 110(3), 346-359.

Benetazzo, A., Gamba, M. and Barbariol, F., 2017. Unseeded large scale PIV measurements corrected for the capillarygravity wave dynamics. Rendiconti Lincei, 28(2), 393-404.

Cleveland, W.S., 1979. Robust Locally Weighted Regression and Smoothing Scatterplots. Journal of the American Statistical Association, 74(368), 829-836.

Creutin, J.D., Muste, M., Bradley, A.A. and Kim S.C.and Kruger, A., 2003. River gauging using PIV techniques: a proof of concept experiment on the Iowa River. Journal of Hydrology, 277(3), 182-194.

Detert, M., Cao, L. and Albayrak, I., 2019. Airborne Image Velocimetry Measurements at the Hydropower Plant Schiffmühle on Limmat River, Switzerland. HydroSenSoft 2019, 211-217.

Detert, M., Johnson, E.D. and Weitbrecht, V., 2017. Proof-ofconcept for low-cost and non-contact synoptic airborne river flow measurements. International Journal of Remote Sensing, 38(8-10), 2780-2807.

Detert, M. and Weitbrecht, V., 2015. A low-cost airborne velocimetry system: proof of concept. Journal of Hydraulic Research, 53(4), 532-539.

Fujita, I. and Hino, T., 2003. Unseeded and seeded PIV measurements of river flows videotaped from a helicopter. Journal of Visualization, 6(3), 245-252.

Fujita, I. and Kunita, Y., 2011. Application of aerial LSPIV to the 2002 flood of the Yodo River using a helicopter mounted high density video camera. Journal of Hydro-environment Research, 5(4), 323-331.

Fujita, I., Muste, M. and Kruger, A., 1998. Large-scale particle image velocimetry for flow analysis in hydraulic engineering applications. Journal of Hydraulic Research, 36(3), 397-414.

Fujita, I., Notoya, Y. and Shimono, M., 2015. Development of UAV-based river surface velocity measurement by STIV based on high-accurate image stabilization techniques. E-proceedings of the 36th IAHR World Congress. 28.
Ioli, F., 2020. Evaluation of Airborne Image Velocimetry approaches using low-cost UAVs in riverine environments. Master thesis. Politecnico di Milano.

Jodeau, M., Hauet, A., Le Coz, J. and Bodart, G., 2019. FudaaLSPIV Version 1.7.1 User Manual. https : //forge . irstea. $\mathrm{fr} /$ attachments/download/2865 (visited on 16/04/2020).

Jodeau, M., Hauet, A., Paquier, A., Le Coz, J. and Dramais, G., 2008. Application and evaluation of LS-PIV technique for the monitoring of river surface velocities in high flow conditions. Flow Measurement And Instrumentation, 19(2), 117-127.

Keane, R. and Adrian, R.J., 1992. Theory of cross-correlation analysis of PIV images. Applied Scientific Research, 49(3), 191-215.

Le Coz, J., Hauet, A., Pierrefeu, G., Dramais, G. and Camenen, B., 2010. Performance of image-based velocimetry (LSPIV) applied to flash-flood discharge measurements in Mediterranean rivers. Journal of Hydrology, 394(1-2), 42-52.

Messerli, A. and Grinsted, A., 2015. Image georectification and feature tracking toolbox: ImGRAFT. Geoscientific Instrumentation, Methods and Data Systems, 4(1), 23-34.

Muste, M., Fujita, I. and Hauet, A., 2008. Large-scale particle image velocimetry for measurements in riverine environments. Water Resources Research, 44(4).

Nobach, H., Damaschke, N. and Tropea, C., 2005. Highprecision sub-pixel interpolation in particle image velocimetry image processing. Experiments in Fluids, 39(2), 299-304.

Patalano, A., García, C. and Rodriguez, A., 2017. Rectification of Image Velocity Results (RIVeR): A simple and userfriendly toolbox for large scale water surface Particle Image Velocimetry (PIV) and Particle Tracking Velocimetry (PTV). Computers and Geosciences, 109, 323-330.

Raffel, M., Willert, C. and Kompenhans, J., 1998. Particle image velocimetry: a practical guide. 1st ed. Springer International Publishing.

Raffel, M., Willert, C., Scarano, F., Kähler, C.J., Wereley, S.T. and Kompenhans, J., 2018. Particle image velocimetry: a practical guide. 3rd ed. Springer International Publishing.

Rosten, E. and Drummond, T., 2005. Fusing points and lines for high performance tracking. Tenth IEEE International Conference on Computer Vision. 1, 1508-1515.

Scarano, F., 2002. Iterative image deformation methods in PIV. Measurement Science and Technology, 13(1), R1-R19.

Scarano, F. and Riethmuller, M.L., 1999. Iterative multigrid approach in PIV image processing with discrete window offset. Experiments in Fluids, 26(6), 513-523.

Thielicke, W. and Stamhuis, E., 2014. PIVlab - Towards User-friendly, Affordable and Accurate Digital Particle Image Velocimetry in MATLAB. Journal of Open Research Software, 2(1).

Westerweel, J. and Scarano, F., 2005. Universal outlier detection for PIV data. Experiments in Fluids, 39(6), 1096-1100. 